\title{
Guidelines on Optical Coherence Tomography Angiography Imaging: 2020 Focused Update
}

\author{
Enrico Borrelli - Mariacristina Parravano - Riccardo Sacconi • \\ Eliana Costanzo · Lea Querques · Giovanna Vella · Francesco Bandello • \\ Giuseppe Querques (D)
}

Received: June 22, 2020 / Published online: August 1, 2020

(C) The Author(s) 2020

\begin{abstract}
Optical coherence tomography angiography (OCTA) has significantly expanded our knowledge of the ocular vasculature. In this review, we provide a discussion of the fundamental principles of OCTA and the application of this imaging modality to study the retinal and choroidal vessels. These guidelines are focused on 2020, and include updates since the 2019 publication. Importantly, we will comment on recent findings on OCTA technology with a special focus on the three-dimensional (3D) OCTA visualization.
\end{abstract}

Keywords: Choriocapillaris; Choroid; Image analysis; Optical coherence tomography

Digital Features To view digital features for this article go to https://doi.org/10.6084/m9.figshare.12662402.

E. Borrelli · R. Sacconi - L. Querques · G. Vella ·

F. Bandello · G. Querques ( $₫)$

Ophthalmology Department, San Raffaele

University Hospital, Milan, Italy

e-mail: giuseppe.querques@hotmail.it

M. Parravano - E. Costanzo

IRCCS—Fondazione Bietti, Rome, Italy

G. Vella

Ophthalmology, Department of Surgical, Medical, Molecular Pathology and of Critical Area, University of Pisa, Pisa, Italy angiography; Retinal vessels; Threedimensional

\section{Key Summary Points}

Optical coherence tomography angiography (OCTA) has significantly expanded our knowledge of the ocular vasculature.

While OCTA has a number of advantages, this imaging technique also has limitations that should be appropriately considered during analysis and interpretation of OCTA images.

In this review, we will provide a description of the basic principles of OCTA technology and the ways to obtain OCTA metrics.

Novel applications of OCTA will also be discussed, including the threedimensional visualization.

\section{INTRODUCTION}

Optical coherence tomography angiography (OCTA) has emerged as a noninvasive imaging technique that may rapidly obtain angiographic 
images of the ocular vasculature [1-4]. By performing repeated scans at the same retinal location, OCTA is indeed able to detect the movement of blood. The result is that OCTA may visualize the retinal and choroidal vasculature at specific depths. The retinal vasculature may be divided into three distinct retinal capillary layers: the superficial capillary plexus (SCP), middle capillary plexus (MCP), and deep capillary plexus (DCP) (Fig. 1) [5, 6]. Furthermore, the radial peripapillary capillary plexus (RPCP) is usually considered as a separate retinal vascular layer as it mainly nourishes the superficial nerve fiber layer (NFL) around the optic disc. In addition, OCTA is effective in visualizing the innermost part of the choroid which is termed the choriocapillaris (CC).

While OCTA has a number of advantages, this imaging technique also has limitations that should be appropriately considered during analysis and interpretation of OCTA images [1].

In this review, we will provide a description of the basic principles of OCTA technology and the ways to obtain OCTA metrics. In addition, we will discuss recent novel applications of this imaging modality. Therefore, this review is aimed at guiding scientists and clinicians on the better interpretation of OCTA findings. Of note, these guidelines are focused on 2020, and include updates since the 2019 publication [3]. This article is based on previously conducted studies and does not contain any studies with human participants or animals performed by any of the authors.

\section{OPTICAL COHERENCE TOMOGRAPHY ANGIOGRAPHY}

\section{Overview on Technical Aspects}

OCTA devices execute repeated B scans at the same location and the obtained structural data are compared to identify signal modifications due to flowing erythrocytes (motion contrast). Importantly, each B scan comprises different A scans which are captured at following locations to finally obtain the B scan. The A scan rate differs among instruments and it is typically includes between 70,000 and 100,000 A scans/s (spectral and swept source devices, respectively). Of note, OCTA research devices may have faster acquisition speeds.

Finally, OCTA data may be captured using either spectral domain OCT (SD OCT) or sweptsource OCT (SS OCT).

The interscan time has a crucial role in OCTA imaging and this parameter represents the time interval between two repeated B scans at the same location. The speed of commercially available OCT systems is up to $100 \mathrm{kHz}$, but experimental OCT systems have pushed into megahertz A scan rates [7]. Importantly, as a shorter interscan time may reduce sensitivity to motion, this also decreases the incidence of motion artifacts that may confound the motion signal [8]. The interscan time and the background noise determine the slowest detectable flow [3]. In addition, the interscan time is also associated with the maximum scan width.

Three distinct methodologies may be employed to detect motion contrast using OCTA devices, as follows: (i) phase-based; (ii) amplitude-based; and (iii) complex amplitudebased, in which OCTA algorithms use both amplitude and phase information.

Currently, commercial instruments and most OCTA studies are limited in the assessment of vascular patterns and organization, without the ability to measure vascular velocity. Experimental devices have been implemented with complex algorithms that allow one to quantify the flow velocity $[9,10]$.

\section{Visualization of OCTA Images}

While OCTA data may be viewed using both two-dimensional (2D) B scan and en face images, the latter visualization is often preferred. To obtain 2D en face images, the volumetric OCTA scan is segmented at specific depths and the flow data within any slab are summed or projected into a $2 \mathrm{D}$ en face image that can be visualized and studied (Fig. 1).

The generation of 2D OCTA en face images may be obtained using different methods, as follows: 



Fig. 1 Optical coherence tomography angiography image of the macula of a healthy subject. The retinal vascularization at the macula includes four different plexuses: the superficial (SCP, first line from top), middle (MCP, second from top), deep (DCP, third from bottom) retinal capillary plexuses, and choriocapillaris (CC, bottom). OCTA images are mainly displayed with en face visualization (images on the right) which is obtained by segmenting the volumetric OCTA scans at specific depths (indicated with the red boundaries). Using this strategy, the flow data within any slab, whose boundaries are red in the left images, are summed or projected into a two- dimensional en face image that can be viewed and studied. These boundaries follow pre-defined layers which can be differentiated on the basis of reflectivity, texture, or other attributes. The layers are (i) the inner limiting membrane (ILM), (ii) the inner border of the inner plexiform layer (IPL), (iii) the outer border of the outer plexiform layer (OPL), and (iv) Bruch's membrane (BM). This image was captured using the Spectralis HRA + OCT imaging device with OCT Angiography Module (Heidelberg Engineering, Heidelberg, Germany) 
- The "maximal intensity projection" approach works by projecting the maximum intensity pixel within each column. This algorithm may be more sensible in detecting smaller vessels. However, it may also be characterized by more noise.

- The "average intensity projection" works by projecting the average intensity pixel within each column. This algorithm is less susceptible to noise but may be less sensitive for small vessels.

A recent study by Byon and colleagues [11] evaluated the impact of the algorithms to obtain the 2D en face CC images on the repeatability of OCTA metrics. The latter study demonstrated that en face CC OCTA images generated using the maximal intensity projection had the highest repeatability.

Of note, the en face visualization of the OCTA images is susceptible to segmentation artifacts, especially in eyes with retinal and choroidal disorders [12]. Therefore, a three-dimensional (3D) visualization has recently been suggested as a more appropriate visualization for OCTA data (see Sect. "Three-Dimensional OCTA" for further details).

OCTA flow information may also be visualized on B scan images. In this visualization, OCTA flow data is typically depicted within cross-sectional OCT data by a pseudocolor overlay on the grayscale structural OCT image.

\section{Limitations and Artifacts}

OCTA images may be significantly impacted by low signal strength. Several factors may influence signal strength, including media opacities. The ratio between the signal, which constitutes the information component dependent on the imaged tissue, and the non-signal component (or noise) is termed the signal to noise ratio and represents a mathematical relationship that may significantly influence the image quality. A decreased signal to noise ratio causes an adjustment of the grayscale range with a consequent increase in image noise and artifact-related OCTA signal. Averaging of multiple en face OCTA images may reduce noise and improve vessels' continuity, as well as significantly ameliorate qualitative and quantitative measurements $[13,14]$. False OCTA signals may be also generated by patient movements [15]. However, eye tracking systems have significantly decreased the impact of these artifacts in OCTA imaging.

Segmentation errors represent a significant and recurring OCTA artifact $[12,15,16]$. As discussed above, en face OCTA images are displayed by locating two boundaries throughout the retinal and/or choroidal structure, and vessels are therefore visualized using different available algorithms. These boundaries use predefined layers identified on structural OCT images. Segmentation errors are common in pathological conditions [12]. As an example, eyes with diabetic macular edema are frequently characterized by segmentation errors, as recently demonstrated by Ghasemi Falavarjani and colleagues [16]. A recent study using convolutional neural networks (CNNs) has demonstrated the utility of this algorithm to improve segmentation of OCTA data [17].

Other artifacts include projection (decorrelation tails) and shadowing artifacts that affect the visualization of the vascular layers. While projection artifacts are secondary to transmitted fluctuating light reaching the deeper retinal layers or the choroid [18-20], shadowing artifacts occur when the OCT beam is attenuated or blocked, thereby impeding its passage to the deeper layers of the retina/choroid [2].

\section{OCTA Quantitative Metrics for Retinal Vessels}

OCTA en face images comprise pixels that may be in a grayscale range of possible values from 0 to $255[2,3,8]$. In order to obtain OCTA metrics, these images are processed with a threshold that is applied to create binary images-in which pixels with a grayscale value above the applied threshold are displayed as white (or black) and pixels falling under the threshold are displayed as black (or white, respectively) from grayscale images $[2,3,8]$. Three different methodologies may be used to binarize OCTA images, as follows: (i) global thresholding; (ii) local thresholding; and (iii) complex 
thresholding, in which binarization is obtained by employing both global and local thresholding algorithms. Of note, while a global threshold applies the same threshold value across the entire image, local (or adaptive) algorithms adapt threshold values to different regions of the image [21]. Additionally, the binarized OCTA images may be skeletonized, the latter process aimed at obtaining an image in which vessels are displayed as one-pixel-width tracings [22].

Binarized and skeletonized OCTA images may be employed to obtain different quantitative metrics:

- The perfusion density can be calculated as a unitless proportion of the number of pixels over the threshold divided by the total number of pixels in the analyzed area on the binarized image.

- The vessel length density is defined as the total length of the perfused vasculature divided by the total number of pixels in the analyzed area on the skeletonized image.

- The vessel diameter index, which represents the average vessel caliber, may be calculated by dividing the total vessel area in the binarized image by the total vessel length in the skeletonized image [23].

- The fractal dimension, which is a mathematical parameter that describes the complexity of a biological structure [24].

Although OCTA quantitative metrics have been widely used in previous studies, this quantification is not without challenges. As an example, quantifiable metrics obtained using different OCTA devices were proved to be not homogeneous [25] and that measurements are strictly dependent on the OCTA scan size [26]. More importantly, differences in binarization thresholding methodologies were proved to significantly influence the final quantification of OCTA metrics in healthy eyes [27, 28]. Given these aspects, there is little consistency in the methodology engaged to calculate OCTA quantitative metrics. These methodologies are even more complicated assuming that OCTA en face images may be also processed with adjustments in brightness and/or contrast (histogram adjustments) before being binarized. As with binarization thresholding, there is no agreement on the best methodology for modifying image histograms.

\section{OCTA Quantitative Metrics for Choriocapillaris}

Flow voids (also known as signal voids) are small dark areas that characterize OCTA images of the CC and are thought to constitute CC vascular dropout or flow reduction. Flow voids have been fully characterized in healthy and pathologic conditions [2, 29-35].

In order to quantify the CC perfusion, en face OCTA images of this vascular layer may be binarized to separate pixels constituting these images into two groups: one that represents the vasculature and another representing flow deficits (FDs). Although a global threshold may be used to obtain binarized images of the $\mathrm{CC}$, this approach may be problematic in the presence of pathologic conditions (e.g., drusen, intraretinal exudates) that may cause local changes in image brightness that may be erroneously identified as flow voids [31, 32, 34, 36, 37]. In order to mitigate this issue, most previous studies adopted a local threshold (Phansalkar method) which uses a small window rather than the whole image to determine the threshold for binarization. A recent study by $\mathrm{Chu}$ and colleagues [38] assessed the proper use of this threshold for CC binarization. They demonstrated that it is important to apply a proper window radius for analyzing OCTA images of the CC, which is strictly dependent on the scan size.

\section{Three-Dimensional OCTA}

As discussed above, while OCTA data are mainly displayed using 2D en face images, recent evidence suggests that a $3 \mathrm{D}$ analysis may permit a reliable visualization and quantification of the retinal vessels (Figs. 2 and 3) [39-43].

First, 2D OCTA images are limited in displaying anatomical entities where both their en face extent and depth information are important to better understand their characteristics, such as type 3 macular neovascularization $(\mathrm{MNV})$ in age-related macular degeneration 


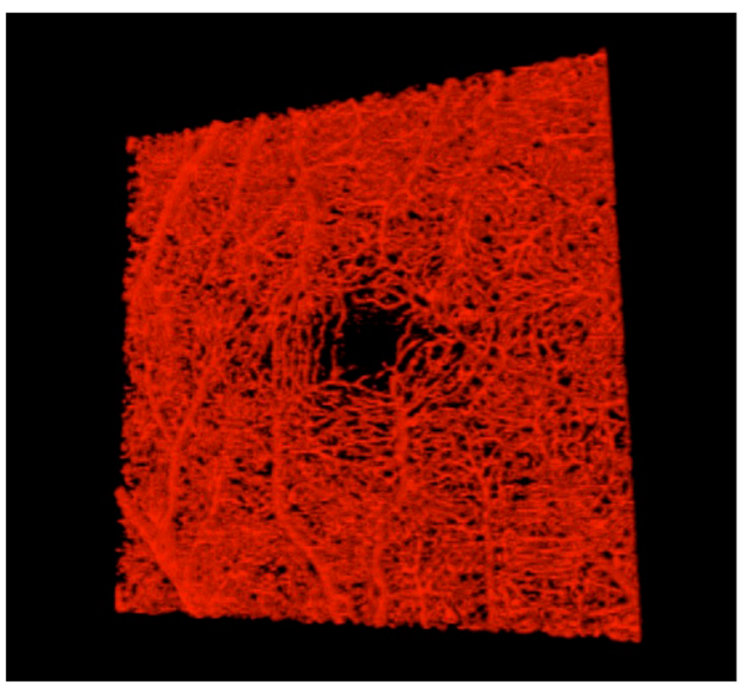

Fig. 2 Optical coherence tomography angiography scans $(3 \times 3 \mathrm{~mm})$ of the macula of a healthy subject (left) and a patient with diabetes (right). Three-dimensional (3D) OCTA images were obtained after thresholding postprocessing 3D OCTA data. Voxels above the applied

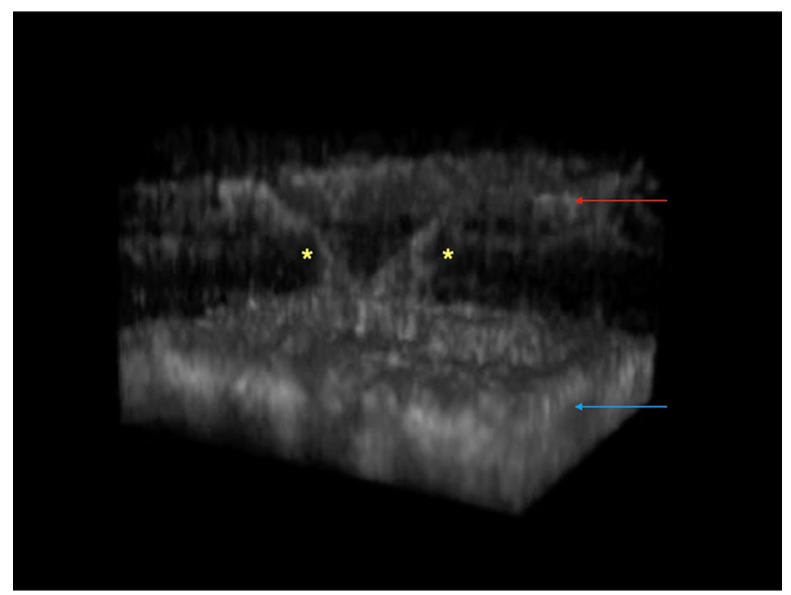

Fig. 3. 3D optical coherence tomography angiography visualization of a macular type 3 neovascularization associated with age-related macular degeneration revealed the presence of two distinct intraretinal lesions with a filiform shape (marked with asterisks), which originate from the deep vascular complex (red arrow) and develop toward the choroid (blue arrow)

(Fig. 3) and microaneurysms in diabetes. These shortcomings may be partially addressed with the development of 3D illustrations, assuming that the acquired OCTA volume enables 3D

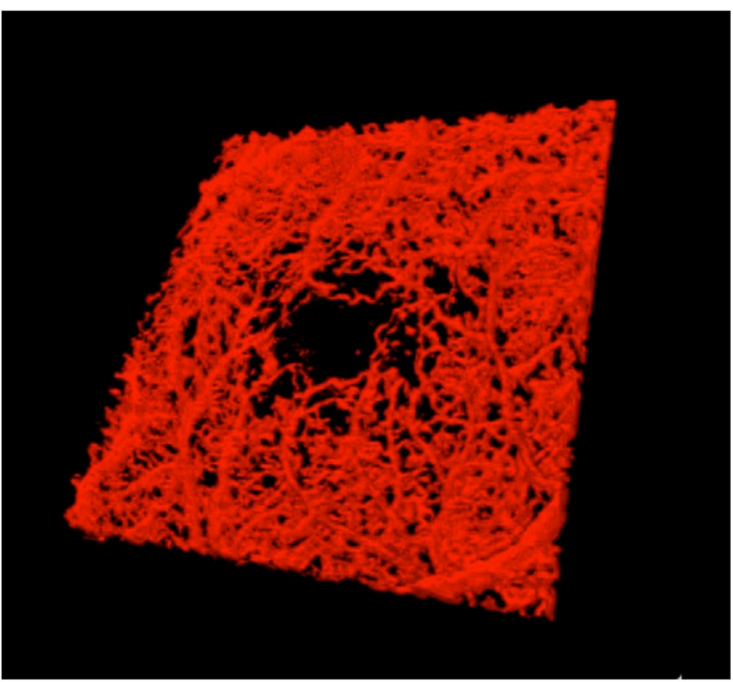

threshold are displayed in red. It is evident that the diabetic case is characterized by a reduced retinal perfusion, as compared with the healthy case

visualization of the retinal and choroidal microvasculature.

We described rotational 3D visualizations of type $3 \mathrm{MNV}$ [42] and diabetic microaneurysms [41] in detail and this approach was demonstrated to be useful for accurate characterization of these vascular malformations. In the evaluation of type $3 \mathrm{MNV}$, our study demonstrated that the 3D visualization allowed one to identify more lesions emerging from the deep vascular complex, as compared with the standard $2 \mathrm{D}$ visualization. Assuming that conventional en face and B scan OCTA images may be limited by overlapping anatomy and vessel foreshortening, results from this study seem to suggest that 3D images may be more appropriate to identify type 3 lesions by resolving these limitations and this visualization may be therefore associated with a higher detection rate [42]. Using the 3D visualization, we also investigated diabetic microaneurysms [41]. This visualization confirmed previous histopathological findings [44], as our study demonstrated that most of the analyzed microaneurysms were associated with two vessels, suggesting no tendency to develop at vascular junctions. Importantly, we were able to identify three 
morphologic patterns featuring microaneurysms. Of note, the "saccular" pattern represented the most common configuration.

Importantly, 2D en face OCTA images may be also limited in the quantification of retinal perfusion as 2D images are obtained by flattening of data in any given segmented volume $[1,3]$. However, this process may result in an underestimation of flow as overlapping vessels in the same slab may appear as merged, and vessels crossing multiple retinal layers and connecting different vascular beds may not be detected [1]. In addition, the 2D OCTA analysis may also overestimate perfusion in cases where retinal vessels or vascular abnormalities (e.g., microaneurysms) cross two consecutive slabs and are thus visualized twice (on two different en face OCTA images) $[41,45]$. Finally, en face images may be significantly affected by segmentation errors, especially in pathological conditions $[1,16]$.

Conversely, a 3D illustration does not require flattening of flow data and it is independent of data segmentation [41, 42]. More importantly, we recently introduced two novel 3D OCTA metrics to quantify macular perfusion (3D vascular volume and 3D perfusion density) [43]. In this previous publication, 15 patients with diabetes and 15 healthy subjects were retrospectively enrolled and their OCTA data were processed for generating 2D and 3D OCTA metrics. Results from the latter study illustrated that 2D and 3D parameters are significantly associated and 3D quantitative metrics differ between patients and controls. In the current study we confirmed that $2 \mathrm{D}$ and $3 \mathrm{D}$ perfusion densities were linearly correlated, although 2D values were significantly higher than $3 \mathrm{D}$ values as $2 \mathrm{D}$ perfusion densities refer to slices of the neuroretina and thus may reasonably overestimate the true perfusion.

Our group recently proposed a quantification of the 3D retinal vasculature in healthy subjects and patients with diabetes [43]. We adopted a standardized methodology to obtain $3 \mathrm{D}$ visualization of the retinal circulation in the macular region and we also introduced two novel 3D quantitative metrics to measure macular perfusion in diabetic retinopathy and healthy eyes: (i) 3D vascular volume and (ii) 3D perfusion density [43]. In the latter study, we demonstrated that this approach may be effective to provide quantitative data from the macular microvascular networks. Importantly, our results illustrated that $2 \mathrm{D}$ and 3D parameters are significantly associated and 3D quantitative metrics differ between patients and controls.

\section{EVALUATION OF VASCULAR LAYERS WITH OCTA}

\section{Retinal Vessels}

OCTA has significantly expanded our knowledge on the organization of the retinal vessels. The retinal vascularization may be separated into the following plexuses: the superficial capillary plexus (SCP), the middle (or intermediate) capillary plexus (MCP), the deep capillary plexus (DCP), and the radial peripapillary capillary plexus (RPCP) [46-49].

In their recent OCTA study of normal subjects, Chanwimol et al. [50] established the retinal vessel quantities for specific vascular layers along the fovea-optic nerve axis. The authors demonstrated a moderate correlation between retinal vessel quantity and structural retinal layer thickness. For the nerve fiber layer, the vessel quantity was highest nasally and declined towards the fovea. Considering the other slabs, the highest vessel quantity was found in the parafoveal region present in the ganglion cell layer.

Around the foveal avascular zone (FAZ), the retinal capillary plexuses converge to form a single parafoveal capillary loop and collectively define the borders of the FAZ [48]. The FAZ area was demonstrated to increase in size with age $[51,52]$ and disease (e.g., myopia, hypertension) $[53,54]$.

\section{Choriocapillaris}

OCTA is a key technology in the evaluation of the $\mathrm{CC}$, assuming that dye angiography techniques have significant limitations for the evaluation of the CC $[2,29,30]$. 
En face OCTA images of the CC are generated by segmenting a thin slab with a thickness of $10-30 \mu \mathrm{m}$ starting at Bruch's membrane. As discussed above, OCTA CC en face images are characterized by small dark regions (flow voids) $[55,56]$ that alternate with granular bright areas, the latter thought to represent CC flow [56]. Please refer to our previous review for further details [3].

\section{CONCLUSIONS}

OCTA is a rapidly evolving technology that has significantly improved the visualization of the retinal and choroidal vessels. However, major limitations still affect its widespread application. The employment of 3D OCTA visualization might ameliorate our capability to study ocular diseases.

\section{ACKNOWLEDGEMENTS}

Funding. No funding or sponsorship was received for this study or publication of this article.

Authorship. All named authors meet the International Committee of Medical Journal Editors (ICMJE) criteria for authorship for this article, take responsibility for the integrity of the work as a whole, and have given their approval for this version to be published.

Disclosures. Francesco Bandello is a consultant for Alcon (Fort Worth,Texas, USA), Alimera Sciences (Alpharetta, Georgia, USA), Allergan Inc (Irvine, California, USA), Farmila-Thea (Clermont-Ferrand, France), Bayer SheringPharma (Berlin, Germany), Bausch and Lomb (Rochester, New York, USA), Genentech (San Francisco, California, USA), Hoffmann-LaRoche (Basel, Switzerland), Novagali Pharma (Évry, France), Novartis (Basel, Switzerland), Sanofi-Aventis (Paris, France), Thrombogenics (Heverlee, Belgium), Zeiss (Dublin, USA). Giuseppe Querques is a consultant for Alimera Sciences (Alpharetta, Georgia, USA), Allergan Inc
(Irvine, California, USA), Amgen (Thousand Oaks, USA), Bayer Shering-Pharma (Berlin, Germany), Heidelberg (Germany), KBH (Chengdu, China), LEH Pharma (London, UK), Lumithera (Poulsbo, USA), Novartis (Basel, Switzerland), Sandoz (Berlin, Germany), Sifi (Catania, Italy), Sooft-Fidea (Abano, Italy), Zeiss (Dublin, USA). Mariacristina Parravano is a consultant for Allergan Inc (Irvine, California, USA), Bayer Shering-Pharma (Berlin, Germany), Novartis (Basel, Switzerland). Giuseppe Querques is also a member of the journal's Editorial Board. Enrico Borrelli, Mariacristina Parravano, Riccardo Sacconi, Eliana Costanzo, Lea Querques and Giovanna Vella have nothing to declare.

Compliance with Ethics Guidelines. This article is based on previously conducted studies and does not contain any studies with human participants or animals performed by any of the authors.

Open Access. This article is licensed under a Creative Commons Attribution-NonCommercial 4.0 International License, which permits any non-commercial use, sharing, adaptation, distribution and reproduction in any medium or format, as long as you give appropriate credit to the original author(s) and the source, provide a link to the Creative Commons licence, and indicate if changes were made. The images or other third party material in this article are included in the article's Creative Commons licence, unless indicated otherwise in a credit line to the material. If material is not included in the article's Creative Commons licence and your intended use is not permitted by statutory regulation or exceeds the permitted use, you will need to obtain permission directly from the copyright holder. To view a copy of this licence, visit http://creativecommons.org/licenses/bync/4.0/. 


\section{REFERENCES}

1. Spaide RF, Fujimoto JG, Waheed NK, Sadda SR, Staurenghi G. Optical coherence tomography angiography. Prog Retin Eye Res. 2018;64:1-55.

2. Borrelli E, Sarraf D, Freund KB, Sadda SR. OCT angiography and evaluation of the choroid and choroidal vascular disorders. Prog Retin Eye Res. 2018;1:2.

3. Borrelli E, Sadda SR, Uji A, Querques G. Pearls and pitfalls of optical coherence tomography angiography imaging: a review. Ophthalmol Ther. 2019. https://www.ncbi.nlm.nih.gov/pubmed/30868418. Accessed 12 May 2019.

4. Borrelli E, Sadda SR, Uji A, Querques G. OCT angiography: guidelines for analysis and interpretation. OCT Imaging Cent Nerv Syst Dis. 2020. https://doi.org/10.1007/978-3-030-26269-3_4.

5. Tan PEZ, Yu PK, Balaratnasingam C, et al. Quantitative confocal imaging of the retinal microvasculature in the human retina. Investig Ophthalmol Vis Sci. 2012;53:5728-36.

6. Chan G, Balaratnasingam C, Yu PK, et al. Quantitative morphometry of perifoveal capillary networks in the human retina. Investig Ophthalmol Vis Sci. 2012;53:5502-14.

7. Klein T, Wieser W, Eigenwillig CM, Biedermann BR, Huber R. Megahertz OCT for ultrawide-field retinal imaging with a 1050nm Fourier domain modelocked laser. Opt Express. 1050nm;19:3304.

8. Spaide R, Fujimoto JG, Waheed NK, Sadda SR, Staurenghi G. Optical coherence tomography angiography. Prog Retin Eye Res. 2017;2:1.

9. Wei X, Hormel TT, Pi S, Guo Y, Jian Y, Jia Y. High dynamic range optical coherence tomography angiography (HDR-OCTA). Biomed Opt Express. 2019;2:1.

10. Ploner SB, Moult EM, Choi W, et al. Toward quantitative optical coherence tomography angiography: visualizing blood flow speeds in ocular pathology using variable interscan time analysis. Retina. 2016;2:S118-S12626.

11. Byon I, Alagorie AR, Ji Y, Su L, Sadda SR. Optimizing the repeatability of choriocapillaris flow deficit measurement from optical coherence tomography angiography. Am J Ophthalmol. 2020;5:27.

12. Ghasemi Falavarjani K, Al-Sheikh M, Akil H, Sadda SR. Image artefacts in swept-source optical coherence tomography angiography. Br J Ophthalmol. 2016;bjophthalmol-2016-309104. https://bjo.bmj. com/lookup/doi/10.1136/bjophthalmol-2016309104. Accessed 19 Jan 2017.

13. Uji A, Balasubramanian S, Lei J, Baghdasaryan E, AlSheikh M, Sadda SR. Impact of multiple en face image averaging on quantitative assessment from optical coherence tomography angiography images. Ophthalmology. 2017. https://www.ncbi.nlm. nih.gov/pubmed/28318637. Accessed 7 June 2017.

14. Uji A, Balasubramanian S, Lei J, et al. Multiple enface image averaging for enhanced optical coherence tomography angiography imaging. Acta Ophthalmol. 2018;96:820-7.

15. Spaide RF, Fujimoto JG, Waheed NK. Image artifacts in optical coherence tomography angiography. Retina. 2015;35:2163-80. https://content. wkhealth.com/linkback/openurl?sid=WKPTLP: landingpage\&an=00006982-201511000-00002. Accessed 10 Jan 2017.

16. Ghasemi Falavarjani K, Habibi A, Anvari P, et al. Effect of segmentation error correction on optical coherence tomography angiography measurements in healthy subjects and diabetic macular oedema. Br J Ophthalmol. 2020;104:162-6.

17. Wang J, Hormel TT, Gao L, et al. Automated diagnosis and segmentation of choroidal neovascularization in OCT angiography using deep learning. Biomed Opt Express. 2020;11:927-44.

18. Long AW, Zhang J, Granick S, Ferguson AL. Machine learning assembly landscapes from particle tracking data. Soft Matter. 2015;11:8141-53. https://xlink.rsc.org/?DOI=C5SM01981H

19. Zhang M, Hwang TS, Campbell JP, et al. Projectionresolved optical coherence tomographic angiography. Biomed Opt Express. 2016;7:816. https://www. osapublishing.org/abstract.cfm?URI=boe-7-3-816

20. Patel RC, Wang J, Hwang TS, et al. Plexus-specific detection of retinal vascular pathologic conditions with projection-resolved OCT angiography. Ophthalmol Retin. 2018;2:816-26.

21. Rogowska J. Overview and fundamentals of medical image segmentation. Handb Med Image Process Anal. 2009. https://doi.org/10.1016/B978-0120777 90-7/50009-6.

22. Durbin MK, An L, Shemonski ND, et al. Quantification of retinal microvascular density in optical coherence tomographic angiography images in diabetic retinopathy. JAMA Ophthalmol. 2017;135: 370. https://www.ncbi.nlm.nih.gov/pubmed/ 28301651. Accessed 7 June 2017.

23. Borrelli E, Balasubramanian S, Triolo G, Barboni P, Sadda SR, Sadun AA. Topographic macular 
microvascular changes and correlation with visual loss in chronic Leber hereditary optic neuropathy. Am J Ophthalmol. 2018;192:217-28. https://www. ncbi.nlm.nih.gov/pubmed/29885298. Accessed 17 July 2018.

24. Zahid S, Dolz-Marco R, Freund KB, et al. Fractal dimensional analysis of optical coherence tomography angiography in eyes with diabetic retinopathy. Investig Ophthalmol Vis Sci. 2016;2:1.

25. Corvi F, Pellegrini M, Erba S, Cozzi M, Staurenghi G, Giani A. Reproducibility of vessel density, fractal dimension, and foveal avascular zone using 7 different optical coherence tomography angiography devices. Am J Ophthalmol. 2018;186:25-31.

26. Rabiolo A, Gelormini F, Marchese A, et al. Macular perfusion parameters in different angiocube sizes: does the size matter in quantitative optical coherence tomography angiography? Investig Ophthalmol Vis Sci. 2018;59:231-7.

27. Rabiolo A, Gelormini F, Sacconi R, et al. Comparison of methods to quantify macular and peripapillary vessel density in optical coherence tomography angiography. PLoS One. 2018;13: e0205773. https://www.ncbi.nlm.nih.gov/pubmed/ 30335815. Accessed 12 Dec 2018.

28. Mehta N, Liu K, Alibhai AY, et al. Impact of binarization thresholding and brightness/contrast adjustment methodology on optical coherence tomography angiography image quantification. Am J Ophthalmol. 2019;205:54-655.

29. Sacconi R, Borrelli E, Corbelli E, et al. Quantitative changes in the ageing choriocapillaris as measured by swept source optical coherence tomography angiography. Br J Ophthalmol. 2018; bjophthalmol2018-313004. https://www.ncbi.nlm.nih.gov/ pubmed/30361273. Accessed 7 Dec 2018.

30. Nassisi M, Baghdasaryan E, Tepelus T, Asanad S, Borrelli E, Sadda SR. Topographic distribution of choriocapillaris flow deficits in healthy eyes. PLoS One. 2018;13:638.

31. Borrelli E, Souied EH, Freund KB, et al. Reduced choriocapillaris flow in eyes with type 3 neovascularization due to age-related macular degeneration. Retina. 2018;38:1968-76.

32. Borrelli E, Uji A, Sarraf D, Sadda SR. Alterations in the choriocapillaris in intermediate age-related macular degeneration. Investig Opthalmology Vis Sci. 2017;58:4792-8. https://www.ncbi.nlm.nih. gov/pubmed/28973325. Accessed 4 Oct 2017.

33. Borrelli E, Mastropasqua R, Senatore A, et al. Impact of choriocapillaris flow on multifocal electroretinography in intermediate age-related macular degeneration eyes. Investig Ophthalmol Vis Sci. 2018;59:25-50.

34. Borrelli E, Palmieri M, Viggiano P, Ferro G, Mastropasqua R. Photoreceptor damage in diabetic choroidopathy. Retina. 2019. https://www.ncbi. nlm.nih.gov/pubmed/30986798. Accessed 13 Jun 2019.

35. Borrelli E, Uji A, Toto L, Viggiano P, Evangelista F, Mastropasqua R. In vivo mapping of the choriocapillaris in healthy eyes: a widefield swept source optical coherence tomography angiography study. Ophthalmol Retin. 2019;2:1.

36. Borrelli E, Shi Y, Uji A, Balasubramanian S, Sarraf D, Sadda SR. Topographical analysis of the choriocapillaris in intermediate age-related macular degeneration. Am J Ophthalmol. 2018;196:34-433.

37. Borrelli E, Mastropasqua R, Senatore A, et al. Impact of choriocapillaris flow on multifocal electroretinography in intermediate age-related macular degeneration eyes. Investig Opthalmology Vis Sci. 2018;59:AMD25. https://www.ncbi.nlm.nih. gov/pubmed/29860309. Accessed 9 July 2018.

38. Chu Z, Cheng Y, Zhang Q, et al. Quantification of choriocapillaris with Phansalkar's local thresholding: pitfalls to avoid. Am J Ophthalmol. 2020. https://linkinghub.elsevier.com/retrieve/pii/ S0002939420300568. Accessed 18 Feb 2020.

39. Spaide RF. Volume rendering of optical coherence tomography angiography reveals extensive retinal vascular contributions to neovascularization in ocular toxoplasmosis. Retina. 2015;35:11.

40. Spaide RF, Suzuki M, Yannuzzi LA, Matet A, BeharCohen F. Volume-rendered angiographic and structural optical coherence tomography angiography of macular telangiectasia type 2 . Retina. 2017;37:424-35.

41. Borrelli E, Sacconi R, Brambati M, Bandello F, Querques G. In vivo rotational three-dimensional OCTA analysis of microaneurysms in the human diabetic retina. Sci Rep. 2019;9:167.

42. Borrelli E, Sacconi R, Klose G, de Sisternes L, Bandello F, Querques G. Rotational three-dimensional OCTA: a notable new imaging tool to characterize type 3 macular neovascularization. Sci Rep. 2019;9: 170.

43. Borrelli E, Sacconi R, Querques L, Battista M, Bandello F, Querques G. Quantification of diabetic macular ischemia using novel three-dimensional optical coherence tomography angiography metrics. J Biophotonics. 2020;11:152. 
44. Moore J, Bagley S, Ireland G, McLeod D, Boulton ME. Three dimensional analysis of microaneurysms in the human diabetic retina. J Anat. 1999;194: 89-100.

45. Hasegawa N, Nozaki M, Takase N, Yoshida M, Ogura Y. New insights into microaneurysms in the deep capillary plexus detected by optical coherence tomography angiography in diabetic macular edema. Investig Ophthalmol Vis Sci. 2016;57: 348-55.

46. Savastano MC, Lumbroso B, Rispoli M. In vivo characterization of retinal vascularization morphology using optical coherence tomography angiography. Retina. 2015;35:2196-203.

47. Garrity ST, Paques M, Gaudric A, Freund KB, Sarraf D. Considerations in the understanding of venous outflow in the retinal capillary plexus. Retina. 2017;37:1809.

48. Campbell JP, Zhang M, Hwang TS, et al. Detailed vascular anatomy of the human retina by projection-resolved optical coherence tomography angiography. Sci Rep. 2017;7:42201.

49. Nesper PL, Fawzi AA. Human parafoveal capillary vascular anatomy and connectivity revealed by optical coherence tomography angiography. Investig Ophthalmol Vis Sci. 2018;59:3858.

50. Chanwimol K, Hirano T, Bedolla A, et al. Evaluation of retinal vessel quantity within individual retinal structural layers using optical coherence tomography angiography. Graefes Arch Clin Exp Ophthalmol. 2020;1-6. https://link.springer.com/10.1007/ s00417-020-04776-8. Accessed 20 June 2020.
51. Garrity ST, Iafe NA, Phasukkijwatana N, Chen X, Sarraf D. Quantitative analysis of three distinct retinal capillary plexuses in healthy eyes using optical coherence tomography angiography. Investig Ophthalmol Vis Sci. 2017;58:5548.

52. Borrelli E, Lonngi M, Balasubramanian S, et al. Macular microvascular networks in healthy pediatric subjects. Retina. 2018;39:1216-24.

53. Al-Sheikh M, Phasukkijwatana N, Dolz-Marco R, et al. Quantitative OCT angiography of the retinal microvasculature and the choriocapillaris in myopic eyes. Invest Ophthalmol Vis Sci. 2017;58: 2063-9. https://www.ncbi.nlm.nih.gov/pubmed/ 28388703. Accessed 26 Apr 2017.

54. Lee WH, Park J-H, Won Y, et al. Retinal microvascular change in hypertension as measured by optical coherence tomography angiography. Sci Rep. 2019;9:156. https://www.ncbi.nlm.nih.gov/ pubmed/30655557. Accessed 17 Feb 2019.

55. Choi W, Moult EM, Waheed NK, et al. Ultrahighspeed, swept-source optical coherence tomography angiography in nonexudative age-related macular degeneration with geographic atrophy. Ophthalmology. 2015;122:2532-44. https://linkinghub. elsevier.com/retrieve/pii/S0161642015008854. Accessed 20 Jan 2017.

56. Spaide RF. Choriocapillaris signal voids in maternally inherited diabetes and deafness and in pseudoxanthoma elasticum. Retina. 2017;37:2008-14. https://insights.ovid.com/crossref?an=00006982900000000-97141. Accessed 17 May 2017. 